\title{
Eco-Friendly and Facile Synthesis of Antioxidant, Antibacterial and Anticancer Dihydromyricetin- Mediated Silver Nanoparticles
}

This article was published in the following Dove Press journal: International Journal of Nanomedicine

\author{
Zhao $\mathrm{Li} \mathbb{D}^{1,2}$ \\ Iftikhar Ali $\mathbb{D}^{1,3}$ \\ Jiying Qiu ${ }^{4}$ \\ Huanzhu Zhao' \\ Wenya $\mathrm{Ma}^{\mathrm{l}, 2}$ \\ Aiying $\mathrm{Bai}^{5}$ \\ Daijie Wang (1D) \\ Jingchao $\mathrm{Li}^{1}$
}

'Shandong Analysis and Test Center, Qilu University of Technology (Shandong Academy of Sciences), Jinan 2500I4, People's Republic of China; ${ }^{2}$ College of Life Science, Shandong Normal University, Jinan 2500 I4, People's Republic of China; ${ }^{3}$ Department of Chemistry, Karakoram International University, Gilgit 15100, Pakistan; ${ }^{4}$ Institute of Agro-Food Science and Technology, Shandong Academy of Agricultural Sciences, Jinan 250 I00, People's Republic of China; ${ }^{5}$ Jinan Municipal Center for Disease Control and Prevention, Jinan 25000I, People's Republic of China
Correspondence: Daijie Wang; Jingchao Li Shandong Analysis and Test Center, Qilu University of Technology (Shandong Academy of Sciences), Jinan 2500 I4,

People's Republic of China

Tel +86-53182605319

Fax +86-053I-82964889

Email wangdaijie@I26.com;

lijingchao888@sohu.com
Background: Dihydromyricetin (DMY), a natural flavonoid, has reportedly antibacterial, antioxidant, anticancer and other properties. In the present study, DMY was used as a reducing agent and stabilizer to synthesize silver nanoparticles (AgNPs), and the optimal conditions for its synthesis were studied. The DMY-AgNPs were investigated for their DPPH scavenging properties and their potential against human pathogenic and food-borne bacteria viz. Escherichia coli (E. coli), and Salmonella. In addition, DMY-AgNPs also showed excellent inhibitory effects on cancer Hela, HepG2 and MDA-MB-231 cell lines.

Methods: The dihydromyricetin-mediated AgNPs (DMY-AgNPs) were characterized by ultraviolet-visible spectrophotometer (UV-Vis spectra), scanning electron microscopy (SEM), transmission electron microscopy (TEM), dynamic light scattering (DLS), Fourier-transform infrared spectroscopy (FTIR), X-ray photoelectron spectroscopy (XPS), and X-ray powder diffraction (XRD). Antioxidant activity of DMY-AgNPs was determined by 1.1-diphenyl-2-picrylhydrazyl (DPPH) scavenging. The antibacterial activity was determined by 96-well plate (AGAR) gradient dilution, while anticancer potential was determined by MTT assay.

Results: The results showed that the dispersion of AgNPs had the maximum UV-visible absorption at about $410 \mathrm{~nm}$. The synthesized nanoparticles were almost spherical. FTIR was used to identify functional groups that may lead to the transformation of metal ions into nanoparticles. The results showed that the prepared AgNPs were coated with biological molecules in the extraction solution. The biosynthesized DMY-AgNPs exhibited good antioxidant properties, at various concentrations $(0.01-0.1 \mathrm{mg} / \mathrm{mL})$, the free radical scavenging rate was about $56-92 \%$. Furthermore, DMY-AgNPs possessed good antibacterial properties against Escherichia coli (E. coli), and Salmonella at room temperature. The minimum inhibitory concentrations (MIC) were $10^{-6} \mathrm{~g} / \mathrm{L}$, and $10^{-4} \mathrm{~g} / \mathrm{L}$, respectively. The bioactivity of DMY-mediated AgNPs was studied using MTT assay against Hela, HepG2 and MDA-MB-231 cancer cell lines, and all showed good inhibitory effects. Conclusion: The present study provides a green approach for the synthesis of DMY-AgNPs which exhibited stronger antioxidant, antibacterial and anticancer properties compared to the dihydromyricetin. DMY-AgNPs can serve as an economical, efficient, and effective antimicrobial material for its applications in food and pharmaceutical fields.

Keywords: natural flavonoid, DMY- $\mathrm{AgNO}_{3}-\mathrm{NPs}$, green approach, DPPH, Escherichia coli, Salmonella, Hela, HepG2, MDA-MB-231

\section{Introduction}

Flavonoids are important natural organic compounds, a kind of secondary metabolites, and have extensive biological activities and pharmacological effects. ${ }^{1}$ Among them, dihydromyricetin (DMY), 3,3',4',5,5',7-hexahydroxy-flavanone has shown 
a wide range of pharmacological effects. ${ }^{2}$ DMY is reported a major component from vine tea (Ampelopsis grossedentata). ${ }^{3}$ It is also extracted from Jujube plant species. DMY has exhibited antioxidant, anti-thrombosis, anti-tumor, and anti-inflammatory activities. ${ }^{4,5}$ In addition to the general properties of flavonoids, DMY has also the properties of relieving alcohol intoxication, preventing alcoholic liver and fatty liver, inhibiting the deterioration of liver cells and reducing the incidence of liver cancer. ${ }^{3}$ Thus, DMY is assumed to be a good product for protecting the liver. Recently DMY has also been reported for its anti-proliferative effect, ${ }^{6}$ protective effect against nonalcoholic steatohepatitis, ${ }^{7}$ atherosclerotic lesion formation inhibitory effect, ${ }^{8}$ antiviral and anti-inflammatory effect, ${ }^{9}$ and a treatment for diabetic cardiomyopathy ${ }^{10}$ etc. However, owing to its lower chemical stability and poor aqueous solubility, despite its medicinal value, DMY usage is limited in clinical, food and pharmaceutical applications. To overcome its solubility issues, as an alternative, different methods are applied.

Nanoparticulate drug delivery systems provide an efficient way for oral bioavailability of different drugs. ${ }^{11}$ Nowadays, the nanoparticles (NPs) are being applied in different fields including biological, electrical, medical and chemical, etc. The size and shape of the NPs are the main factors that determine the performance of NPs. ${ }^{12}$ Silver nanoparticles (AgNPs) are fine particles of metallic silver that have at least one dimension range from 1 to $100 \mathrm{~nm}$. AgNPs are very versatile nanomaterial, famous for their excellent properties as well as for their antimicrobial properties. ${ }^{13}$ Apart from the antibacterial effects, AgNPs have been reported for antifungal and anti-inflammatory properties. AgNPs get attached to the cell membrane and are released in the bacterial cells, which makes them possess good antimicrobial activity. ${ }^{14}$ The AgNPs have exhibited improved antibacterial properties against drugresistant bacteria. ${ }^{15}$

Although there are many ways to synthesize nanoparticles, their green synthesis has been highlighted in recent years. ${ }^{16}$ Green synthesis uses a variety of reductants, including biomass, plant extracts, ${ }^{14,17-19}$ microorganisms ${ }^{20}$ and so on. ${ }^{21,22}$ Among these methods, biomass, especially flavonoids, are considered to be responsible for the reduction of $\mathrm{Ag}^{+}$to AgNPs. Flavonoids possess important application value because of its wide sources and high synthetic efficiency.

In this study, we report a new method for green synthesis of AgNPs, which uses DMY to reduce silver ions into nanoparticles without adding any toxic chemicals. The natural product, DMY, is used as a reducing agent and a stabilizer. Compared with the traditional method, this method is simple, easy to operate, cost-effective and environment friendly. The synthesized DMY-AgNPs were studied for their antioxidant activity using DPPH protocol, and for their antibacterial activity against E. coli, and Salmonella. This study will contribute to the research on further fascinating properties and application of natural biomass in the field of nanomedicine, nanoscience and nanotechnology.

\section{Materials and Methods}

\section{Materials}

DMY was obtained from Chengdu Desite Co., Ltd. (Chengdu, China). 1.1-Diphenyl-2-picrylhydrazyl (DPPH), butylated hydroxytoluene (BHT), silver nitrate, anhydrous alcohol were purchased from Sinopharm Chemical Reagent Co., Ltd (Shanghai, China). All other chemicals, such as trifluoroacetic acid and triethylamine, were of analytical grade and all aqueous solutions were prepared in deionized water. An osmosis Milli-Q system was used to get deionized water.

\section{Apparatus}

Nicolet 710 FT-IR spectrometer (Thermo Nicolet, USA) was employed to analyze the FTIR results. The metal content was determined by IRIS Advantage OPTIMA 7000DV Inductively Coupled Plasma-Atomic Emission Spectrometer (Thermo PerkinElmer). The surface morphology observation, particle size measurement analysis, and scanning electron microscope images of the NPs were determined by SUPRA ${ }^{\mathrm{TM}} 55$ Thermal Field Emission Scanning Electron Microscopy (Carl Zeiss, Germany). The transmission electron microscope images of NPs were studied by JEM-2100F Transmission Electron Microscopy (JEOL, Japan). X-ray diffraction studies were carried out on EMPYREAN X-ray diffractometer (PANalytical, Netherlands). Genesys 10S UV-Vis spectrometer (Thermo Fisher) was used for the ultra-violet and visible spectral analysis. For XPS analysis, Escalab 250xi X-ray photoelectron spectroscopy (Thermo, USA), and for DLS and Zeta potential analysis, NanoBrook Omni (Brookhaven Instruments, USA) were used.

\section{Synthesis of Silver Nanoparticles}

Following the reported protocols ${ }^{23-26}$ the standard sample DMY was accurately weighed and dissolved in $50 \mathrm{~mL}$ of 
$10 \%$ ethanol. Silver nitrate was dissolved in $20 \mathrm{~mL}$ deionized water. Then, $8 \mathrm{~mL}$ DMY solution was taken in $25 \mathrm{~mL}$ round bottom flask, then $2 \mathrm{~mL}$ silver nitrate solution was added to the mixture. Keeping DMY: $\mathrm{AgNO}_{3}$ final molar ratio of $1: 1$, the mixture was put on the oil bath, at a temperature of $50{ }^{\circ} \mathrm{C}$. In 15 minutes, the reaction started, and the reaction mixture becomes a grass green, and visible black particles were suspended in solution, that showed that the AgNPs were generated, after $3 \mathrm{~h}$ of reaction a suspension was formed, which was taken and put in a centrifuge tube. The supernatant was removed after centrifugation at a speed of $8000 \mathrm{rpm}$ for $30 \mathrm{~min}$. The supernatant was retained for the determination of inductively coupled plasma (ICP). The AgNPs were washed with deionized water 2-3 times to remove the excess organic matter. After drying, AgNPs were evenly distributed in water, and kept in the refrigerator for later use.

\section{Optimized Synthesis Conditions}

The optimum conditions for biosynthesis of AgNPs were found by changing the concentration of silver nitrate, $\mathrm{pH}$, and temperature. In order to investigate the effect of silver nitrate concentration, $2 \mathrm{~mL}$ of different concentrations were added to $8 \mathrm{~mL}$ of DMY solution. The molar ratio of DMY and silver nitrate was 2:1, 1:1, 1:2 and 1:4, respectively. In order to explore the possible influence of $\mathrm{pH}$ on the synthesis process, the mixed solutions of trifluoroacetic acid and triethylamine were adjusted with $\mathrm{pH}$ of $2,4,6,7,8,9,10,11$ and 12, respectively. Different temperatures ie $40{ }^{\circ} \mathrm{C}, 50{ }^{\circ} \mathrm{C}, 60{ }^{\circ} \mathrm{C}$ and $70{ }^{\circ} \mathrm{C}$ were also investigated.

\section{Characterization of Silver Nanoparticles Ultraviolet-Visible Spectroscopy}

To determine the development of AgNPs and exploit their optical properties, the surface plasmon resonance (SPR) peaks were monitored by ultraviolet-visible spectrophotometer (UV-Vis spectra). The sampling of aliquots (AgNPs diluted with distilled water) was poured into a $1.0 \mathrm{~cm}$ cuvette. The UV-Vis spectra were recorded at the wavelength range of $330-800 \mathrm{~nm}$ at room temperature.

\section{SEM, TEM, FTIR, XRD, XPS, DLS and Zeta Potential Analysis}

Scanning electron microscopy (SEM) analysis was performed at different magnifications. Images of AgNPs were recorded on a Quanta 200FEG SEM spectrometer at $20 \mathrm{kV}, 10 \mathrm{~mA}$, SE mode and spot size 3.5 (Hillsboro, OR, USA). Prior to observation, the xerogel was sputter coated with a thin layer of gold. Thin films of synthesized and stabilized AgNPs were prepared on a carbon-coated copper grid by just dropping a very small amount of the sample on the grid, and the sample was analyzed for morphology and size of the silver nanoparticles under a mercury lamp for $5 \mathrm{~min}$.

Transmission electron microscopy images of the AgNPs were recorded on a Tecnai G2 20 TWIN transmission electron microscope (Hillsboro) with the support being a copper mesh. The rheology measurements were performed using a Kinexus pro+ Rheometer (Malvern, UK), equipped with a temperature controller and parallel stainless steel plates ( $20 \mathrm{~mm}$ diameter, $0.5 \mathrm{~mm}$ gap). The gel after shaking was placed in the shearing gap of the rheometer and was allowed to incubate at $15{ }^{\circ} \mathrm{C}$. Subsequently it was submitted to a frequency sweep or stress sweep experiment.

The Fourier-transform infrared spectroscopy (FTIR) was studied in the wavelength range between 4000 and $400 \mathrm{~cm}^{-1}$ to find the functional groups present around the synthesized AgNPs. The AgNPs were dried and grinded with $\mathrm{KBr}$ pellets and analyzed.

X-ray powder diffraction (XRD) was carried out by dipping a glass plate loading thin film of the AgNPs in a solution.

The structure of the synthesized DMY-mediated AgNPs was determined using X-ray photoelectron spectroscopy (XPS). The DMY-mediated AgNPs were compacted and then vacuumed for testing.

The particle size was determined using dynamic light scattering (DLS) measurement technique. In addition, zeta potential was used to determine the surface potential of the silver nanoparticles. Liquid samples of the nanoparticles were diluted to a certain concentration by water.

\section{Conversion of $\mathrm{Ag}^{+}$lons}

The synthesized AgNP suspensions were centrifuged at a high speed of $8000 \mathrm{rpm}$ for 30 minutes, and the remaining $\mathrm{Ag}^{+}$was separated from the generated AgNPs. The concentration of $\mathrm{Ag}^{+}$in the supernatant represents the residual or unreacted concentration of $\mathrm{Ag}^{+}$. The reacted $\mathrm{Ag}^{+}$was calculated by deducting the remained $\mathrm{Ag}^{+}$, the $\mathrm{Ag}^{+}$value after reaction was determined by ICP. The conversion rate of $\mathrm{Ag}^{+}$was determined by comparing the mass of $\mathrm{Ag}^{+}$with that of initial $\mathrm{Ag}^{+}$.

\section{Antioxidant Activity of AgNPs}

Following the previous protocols, ${ }^{27,28}$ the antioxidant activity of synthesized nanoparticles was determined by the 
scavenging of 1.1-diphenyl-2-picrylhydrazyl (DPPH) free radicals. DPPH was weighed and dissolved in anhydrous ethanol to produce $0.015 \mu \mathrm{g} / \mathrm{mL}$ solution. Samples of different concentrations were mixed with DPPH to obtain the final concentrations of $0.01,0.25,0.50,0.75,0.10 \mathrm{mg} / \mathrm{mL}$, respectively. Butylated hydroxytoluene (BHT) was used as control. The mixed solution was reacted at $30^{\circ} \mathrm{C}$ for $30 \mathrm{~min}$, and the absorbance of each test tube was determined at 517 $\mathrm{nm}$ through ultraviolet spectrometer. The blank group only contained DPPH. The DPPH free radical scavenging activity was calculated using the following equation.

DPPH scavenging effect $(\%)=\left[\left(A_{0}-A_{t}\right) / A_{0}\right] \times 100$

$A_{0}$ is the absorbance of the control and $A_{t}$ is the absorbance of the sample.

\section{Antibacterial Activity of AgNPs}

The antibacterial activity of AgNPs against human pathogenic bacteria such as E. coli and salmonella was studied following the previous test protocol. ${ }^{29-31}$ The minimum inhibitory concentration (MIC) was obtained by 96-well plate gradient dilution. Under an aseptic condition, AgNPs were mixed in proportion with different bacterial fluids, after adding normal saline to culture for 1 day, a certain amount of liquid was absorbed from the mixture and evenly coated on the AGAR plate containing nutritious broth. After another day of culture at $37{ }^{\circ} \mathrm{C}$, count was carried out after taking out the plate, and finally, an intuitive curve of bacterial inhibition rate was obtained.

\section{Cytotoxicity by MTT Assay}

Hela, HepG2 and MDA-MB-231 cells were seeded into 96-well plate respectively with 5000 cells per each well. After a 48 -hour incubation period, $10 \mu \mathrm{L}$ of a $5 \mathrm{mg} / \mathrm{mL}$ MTT solution was added to each well and the plate was further incubated at $37^{\circ} \mathrm{C}$ for 4 hours. Thereafter, the medium was aspirated and the wells were washed with

PBS, and $150 \mu \mathrm{L}$ of DMSO was added to each well. The plate was placed on a shaker in order to dissolve the dye. After the formazan crystals had dissolved, the absorbance was determined spectrophotometrically at $490 \mathrm{~nm}$ on a microplate reader (PerkinElmer). ${ }^{32}$ The percent inhibition was calculated by the following formula.

Inhibition $(\%)=(\mathrm{OD} 490 \mathrm{~nm}$ value of control well OD $490 \mathrm{~nm}$ value of sample well)/(OD $490 \mathrm{~nm}$ value of control well - OD $490 \mathrm{~nm}$ value of blank well)

\section{Results and Discussion Visible Observation of Synthesized AgNPs}

AgNPs were synthesized using DMY. In the mixing process, the $\mathrm{Ag}^{+}$ions were reduced to AgNPs, which was followed by color change. After being mixed with silver ions, DMY began to change from yellow to purplish red (Figure 1A and B). As the reaction went on, the solution changed from purplish red to yellowish green (Figure 1C), and the formation of black nanoparticles (Figure 1D) was observed.

\section{UV-Visible Spectroscopy Analysis}

As shown in Figure 2A and B the effects of different molar ratios of DMY and silver nitrate on AgNPs synthesis were studied. The molar ratio of DMY and silver nitrate was set as 2:1, 1:1, 1:2 and 1:4. When the molar ratio was $2: 1$, although the silver ion conversion rate was $99.77 \%$, the absorption peak was low. This suggested that DMY overdose affected the size of nanoparticles. When there was more DMY, the silver ion transformation was relatively complete, but the excessive amount of DMY affected the size of nanoparticles, making the peak deformation shorter and wider. When the molar ratio was $1: 2$ and 1:4, silver nitrate was not completely transformed, the silver ion conversion rate was only
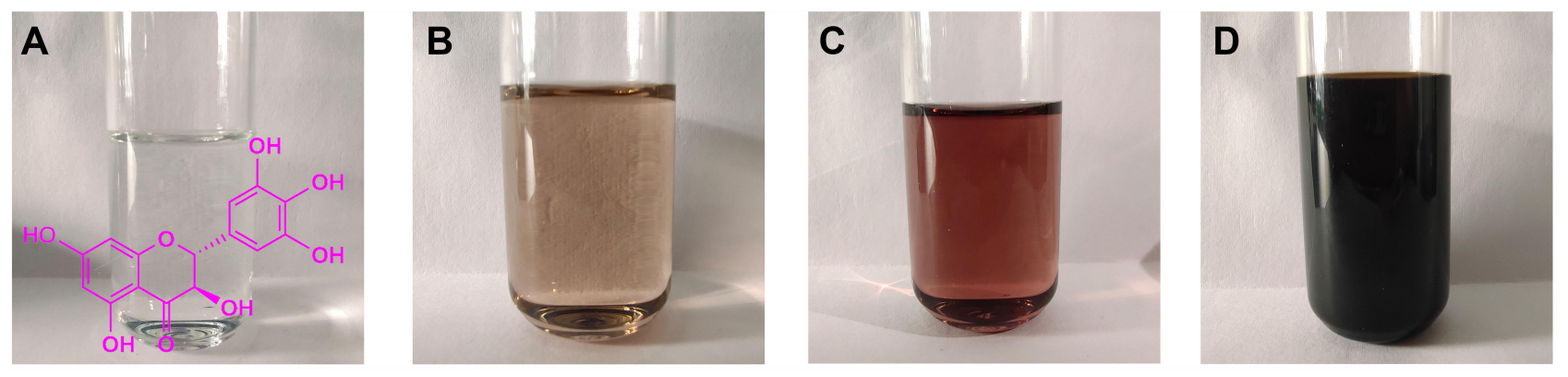

Figure I (A) DMY. (B) DMY after addition of alkali. (C) DMY is initially mixed with $\mathrm{AgNO}_{3}$. (D) DMY reacted with $\mathrm{AgNO}_{3}$ for $3 \mathrm{~h}$ Abbreviations: DMY, dihydromyricetin; $\mathrm{AgNO}_{3}$, silver nitrate. 

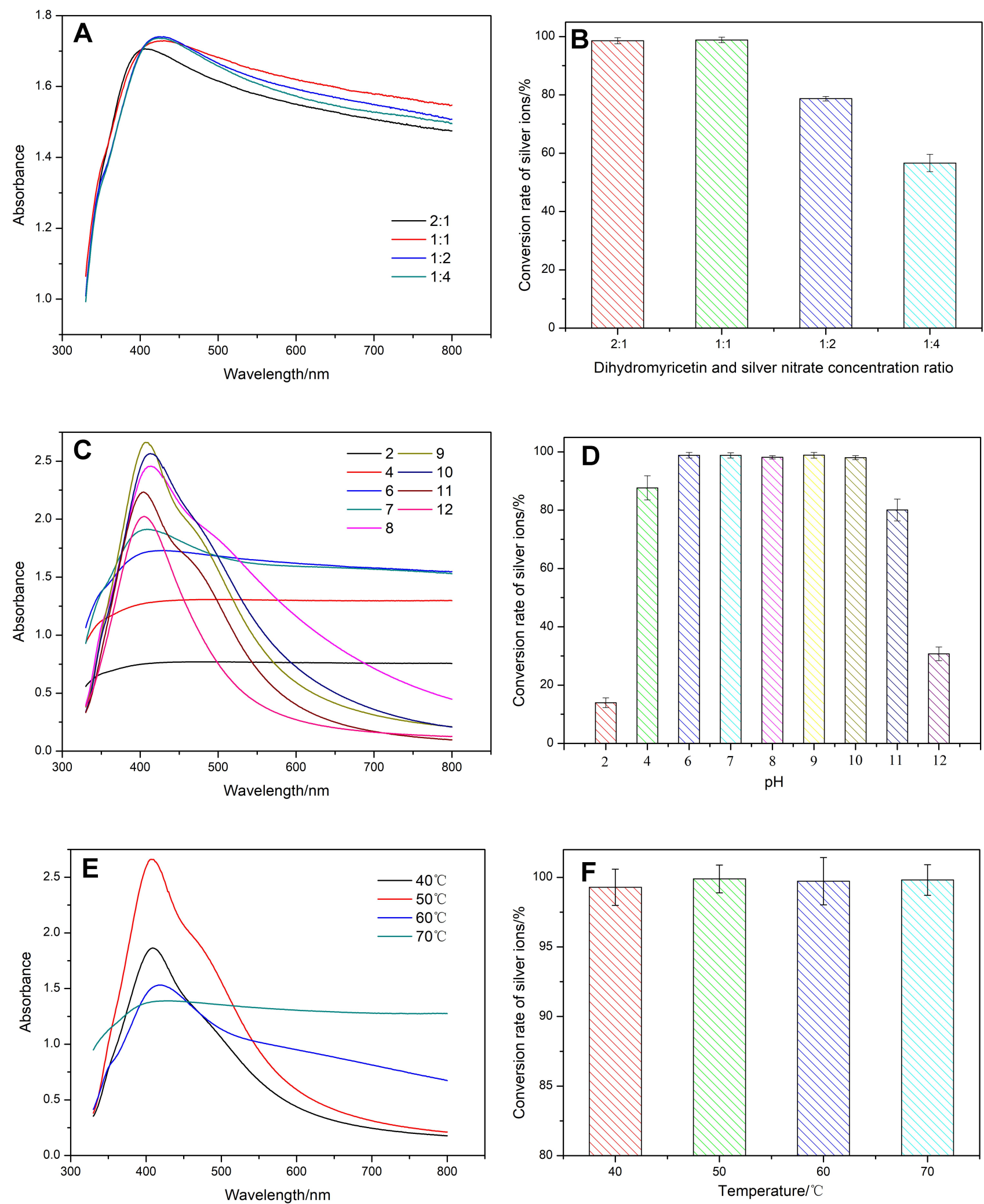

Figure 2 (A) UV-Vis spectra of AgNPs synthesized by DMY at different $\mathrm{AgNO}_{3}$ concentrations. (B) Silver ion conversion rate of $\mathrm{AgNPs}$ synthesized by $\mathrm{DMY}$ at different $\mathrm{AgNO}_{3}$ concentrations. (C) UV-Vis spectra of AgNPs synthesized by DMY at different pH. (D) Silver ion conversion rate of AgNPs synthesized by DMY at different pH. (E) UV-Vis spectra of AgNPs synthesized by DMY at different temperatures. (F) silver ion conversion rate of AgNPs synthesized by DMY at different temperatures.

Abbreviations: UV-Vis, ultraviolet and visible absorption spectroscopy; $\mathrm{AgNPs}$, silver nanoparticles; $\mathrm{DMY}$, dihydromyricetin; $\mathrm{AgNO}_{3}$, silver nitrate; $\mathrm{pH}$, power of concentration of hydrogen ion in solution. 
$79.42 \%$ and $53.21 \%$, respectively. When the molar ratio was $1: 2$, the absorption peak was high and the peak shape was narrow and stable, so it was the optimal molar ratio. Under this condition, the silver ion conversion rate was $99.83 \%$.

Figure $2 \mathrm{C}$ and $\mathrm{D}$ show that the absorption spectra of the mixed solution at different $\mathrm{pH}$ values were recorded. When the mixed solution was acidic, the reaction rate decreased with the decrease of $\mathrm{pH}$ value. It can be explained that the reduction of DMY was affected under strong acidic condition. Therefore, the absorption peaks were relatively wider and the binding rate was lower. When the $\mathrm{pH}$ was 2.0, the silver ion conversion rate was only $12.96 \%$. Under alkaline conditions, the absorption peaks of the solution became higher and narrower. However, at $\mathrm{pH} 11$ and 12, although the peak shape was good, the silver ion conversion rate was very low, indicating that the strongly alkaline environment was not conducive to the formation of AgNPs. When the $\mathrm{pH}$ was 8 , the peak shape was found the best, and the silver ion conversion rate was $98.89 \%$, so, it was the optimal $\mathrm{pH}$ for the reaction.

Figure $2 \mathrm{E}$ and $\mathrm{F}$ show the effect of different temperatures on AgNPs synthesis. Experiments were carried out under four different reaction temperatures, ie 40, 50, 60, $70{ }^{\circ} \mathrm{C}$. Too low temperature hindered the formation of AgNPs, too high temperature resulted in too fast reaction and agglomeration. Under different temperatures narrow and stable adsorption peaks showed that the best reaction temperature was $50^{\circ} \mathrm{C}$.

To sum up, when the molar ratio of DMY and silver nitrate was 1:1, the optimized conditions for AgNPs synthesis were $\mathrm{pH} \mathrm{9}$, and the reaction temperature was $50{ }^{\circ} \mathrm{C}$.

\section{Characterization}

As shown in Figure 3A, in the SEM analysis, the morphology and size of the synthesized silver nanoparticles of DMY at different magnifications showed circular dotshaped particles. The morphology, size and shape of AgNPs were determined by TEM. The TEM image of AgNPs synthesized under optimized conditions is shown in Figure 3B, and the synthesized AgNPs were found mostly in spherical shape.

FTIR (Figure 3C) was performed to identify the bond linkages and functional groups associated with DMY and $\mathrm{Ag}^{+}$. The results revealed four peaks at 3444, 1630, 1384 and $1117 \mathrm{~cm}^{-1}$. The absorption bands at 3444 and $1630 \mathrm{~cm}^{-1}$ were assigned to the stretching vibration and in- plane bending vibration of $\mathrm{O}-\mathrm{H}$ group, respectively. ${ }^{33}$ The absorption bands at $1630 \mathrm{~cm}^{-1}$ were attributed to the stretching vibration of $=\mathrm{C}-\mathrm{H}$ and $\mathrm{C}=\mathrm{C} .{ }^{34}$ The peak at $1630 \mathrm{~cm}^{-1}$ was assumed to be related to the surface adsorbed water molecule. Moreover, the band at $1117 \mathrm{~cm}^{-1}$ was contributed by the skeletal $\mathrm{C}-\mathrm{O}$ and $\mathrm{C}-\mathrm{C}$ vibration bands of glycosidic linkage. ${ }^{35}$ It indicated the possible presence of the residual flavonoids on the surface of the obtained AgNPs.

The XRD pattern of the synthesized AgNPs is shown in Figure 3D. The narrow peaks in the figure indicated the crystalline properties of the nanoparticles. The XRD pattern showed four intense peaks corresponding to $2 \theta$ values $37.9730^{\circ}, 44.1370^{\circ}, 64.4634^{\circ}$ and $77.3187^{\circ}$ that represented face-centered cubic (fcc) lattice of silver corresponding to the Miller indices of (111), (200), (220), (311), which were similar to the Bragg reflection of silver nanocrystals. According to the intensity ratio of (111) to other diffraction peaks, it was deduced that the (111) plane was the predominant orientation in the silver crystal structure of the biosynthesized AgNPs.

The XPS full spectrum scan pattern of the synthesized AgNPs is shown in Figure 3E. The pattern illustrated that most of the elements in AgNPs were $\mathrm{C}, \mathrm{O}$ and $\mathrm{Ag}$. The results indicated that the surface of silver nanoparticles contains a certain amount of DMY.

The DLS measurement technique was used to evaluate the particle size and surface morphology of AgNPs as prepared. The size distribution of the synthesized AgNPs is depicted in Figure 3F. It was observed that the particles obtained were polydispersed mixtures in the range of 100-230 nm. The average size of the synthesized AgNPs using DMY was around $114.76 \pm 1.34 \mathrm{~nm}$ with PDI $0.301 .^{36,37}$

The zeta potential of the synthesized AgNPs was determined in water as dispersant. The zeta potential was found to be $-16.5 \pm 2.1 \mathrm{mV}$. The high value proved that the DMY-mediated silver nanoparticles have strong stability and dispersibility in water the negative potential value could be due to the possible capping of the DMY. ${ }^{32,37,38}$

\section{Antioxidant Activity}

The free radical scavenging activity of DMY-AgNPs was determined by DPPH method. The scavenging effect of DMY-AgNPs on DPPH free radical is shown in Figure 4. The free radical scavenging activity of DMY-AgNPs was similar to or better than that of BHT. At different concentrations of synthetic silver nanoparticles $(0.01-0.1 \mathrm{mg} /$ $\mathrm{mL}$ ), the free radical scavenging rate was about $56-92 \%$. Recently inclusion of DMY with cyclodextrins ${ }^{39}$ and cookie fortified with $\mathrm{DMY}^{40}$ has been reported for its 

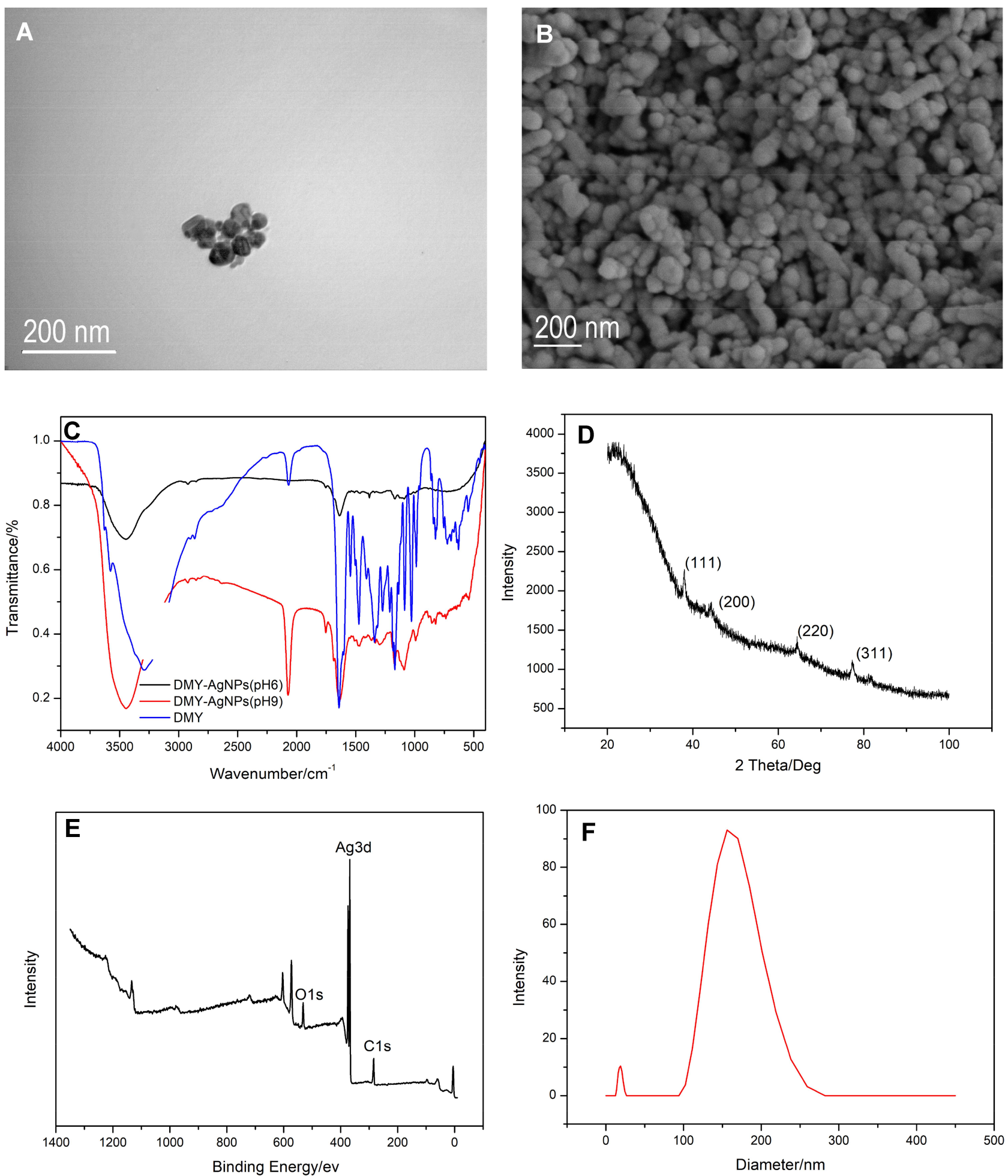

Figure 3 (A) TEM image of DMY-mediated AgNPs. (B) SEM image of DMY-mediated AgNPs. (C) FTIR image of DMY-mediated AgNPs. (D) XRD pattern of DMY-mediated AgNPs. (E) XPS pattern of AgNPs synthesized by DMY (F) DLS pattern of DMY-mediated AgNPs.

Abbreviations: TEM, transmission electron microscopy; DMY, dihydromyricetin; AgNPs, silver nanoparticles; SEM, scanning electron microscopy; FTIR, Fourier-transform infrared spectroscopy; XRD, X-ray powder diffraction; XPS, X-ray photoelectron spectroscopy; DLS, dynamic light scattering. 


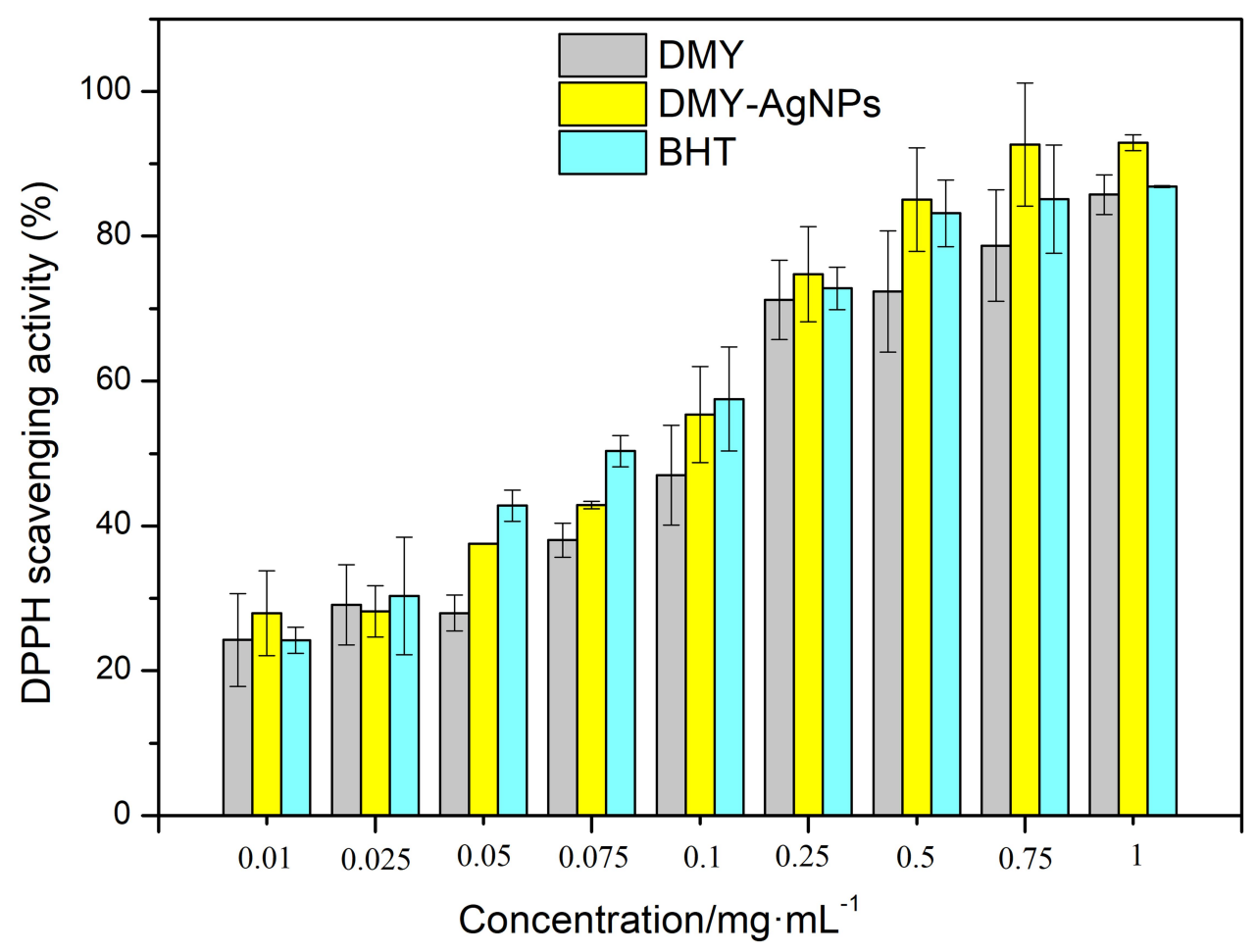

Figure 4 Comparison of free radical DPPH scavenging rate among DMY-AgNPs and DMY and BHT. Abbreviations: DPPH, I.I-diphenyl-2-picrylhydrazyl; DMY-AgNPs, dihydromyricetin-mediated silver nanoparticles; DMY, dihydromyricetin; BHT, butylated hydroxytoluene.

enhanced antioxidant potential. DMY significantly lowered oxidation compared to control in the previous study, ${ }^{3}$ and in the present study, the antioxidant potential was further improved by DMY-mediated AgNPs.

\section{Antibacterial Activity}

Biosynthetic AgNPs significantly inhibited bacterial growth. The minimum inhibitory concentrations (MIC) of AgNPs on E. coli and Salmonella were $10^{-6} \mathrm{~g} / \mathrm{L}$ and $10^{-4} \mathrm{~g} / \mathrm{L}$, respectively, indicating that AgNPs had good inhibitory effects on these two bacteria. Figure 5 shows the amount of live bacteria of culture medium at the same concentration of different samples, which all proved that compared with DMY and tetracycline, DMY-AgNPs have more superior and significant antibacterial effect. Through the inhibition rate curve of $E$. coli and Salmonella (Figure 6), the inhibition rate increased with the increase of sample concentration. By means of bacterial electron microscope (Figure 7), it is clear that the silver nanoparticles damaged the structure and morphology of bacteria to a certain extent, so prevented the growth and reproduction of bacteria. DMY has been reported for its ideal and potent antibacterial activity on Staphylococcus aureus, ${ }^{41,42}$ Bacillus subtilis, E. coli, Salmonella paratyphi, and Pseudomonas aeruginosa. $^{43}$ Recently, DMY-coated multi-walled carbon nanotubes were reported for their antimicrobial properties. ${ }^{44}$ The DMY-mediated AgNPs could also provide an economical and a better alternative for its inhibitory potential against the food-borne bacteria.

\section{Anticancer Activity}

The anticancer potential of DMY-mediated AgNPs was studied using MTT assay against cervical (Hela), liver (HepG2) and breast (MDA-MB-231) cancer cell lines at various concentrations (Figure 8A-C). It was observed that, in the case of all the cell lines, compared to DMY, the DMY-mediated AgNPs showed excellent biocompatibility as expected. For Hela, the AgNPs showed superior continuous effects from low concentrations. When the synthesized AgNPs concentration was $1.5625 \mu \mathrm{g} / \mathrm{mL}$, the inhibition rate reached $28.96 \%$, and it kept increasing with the increase in concentration. For HepG2, the inhibitory effect of DMY-AgNPs was significantly higher than that of DMY in both low and high concentrations. DMY was not particularly effective, suggesting that DMY-mediated AgNPs had a good effect on HepG2 cells. Both DMY and AgNPs showed good inhibitory effect on MDA-MB-231cells. However, AgNPs showed excellent effect at a high concentration, the inhibition rate reached $81.44 \%$ at the highest point. Previously DMY has been reported to enhance anticancer 

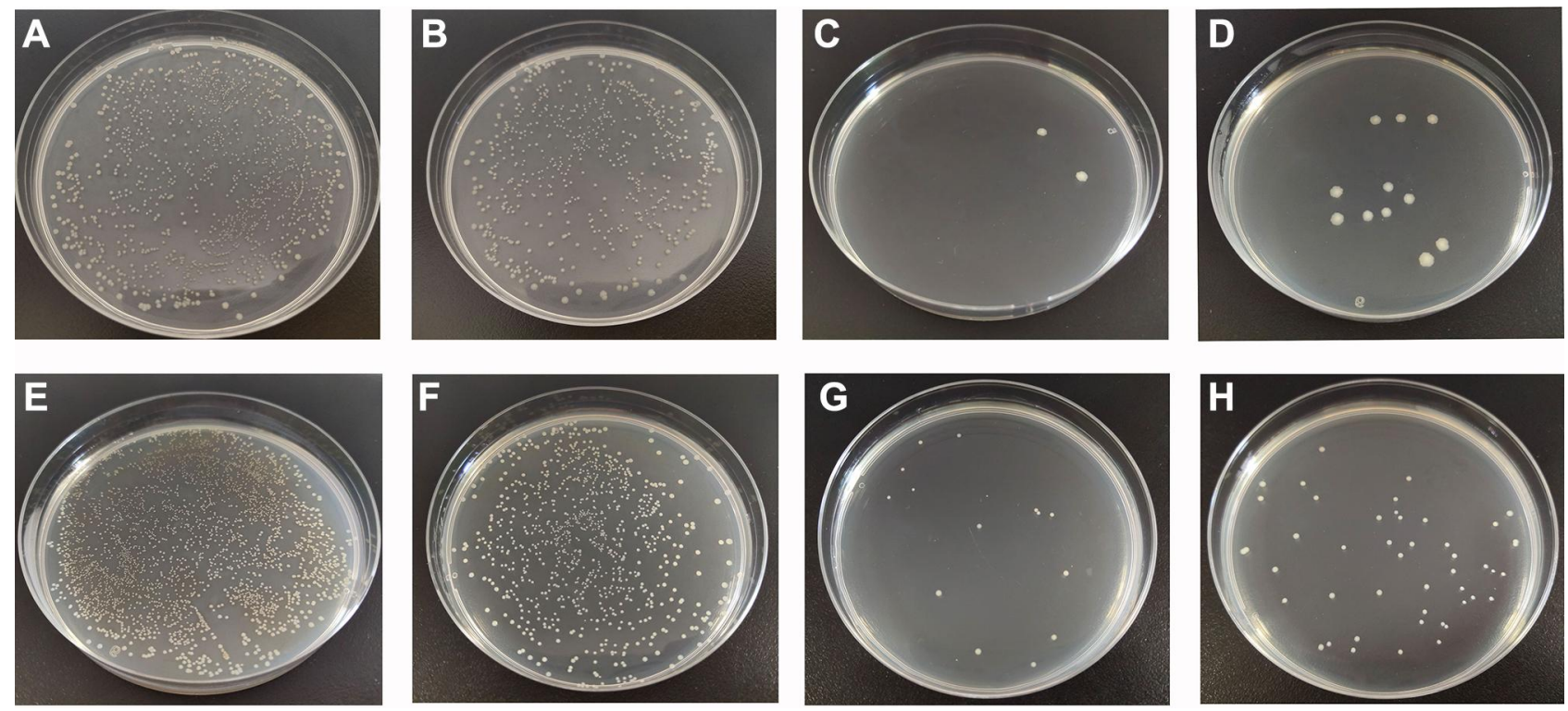

Figure 5 Number of viable bacteria of culture medium. (A) E. coli. (B) E. coli treated with $0.1 \mathrm{mg} / \mathrm{mL}$ DMY. (C) E. coli treated with $0.1 \mathrm{mg} / \mathrm{mL}$ DMY-AgNPs. (D) E. coli treated with $0.1 \mathrm{mg} / \mathrm{mL}$ tetracycline. (E) Salmonella. (F) Salmonella treated with $0.1 \mathrm{mg} / \mathrm{mL}$ DMY. (G) Salmonella treated with $0.1 \mathrm{mg} / \mathrm{mL}$ DMY-AgNPs. (H) Salmonella treated with $0.1 \mathrm{mg} / \mathrm{mL}$ tetracycline.

Abbreviations: E. coli, Escherichia coli; DMY, dihydromyricetin; DMY-AgNPs, dihydromyricetin-mediated silver nanoparticles.
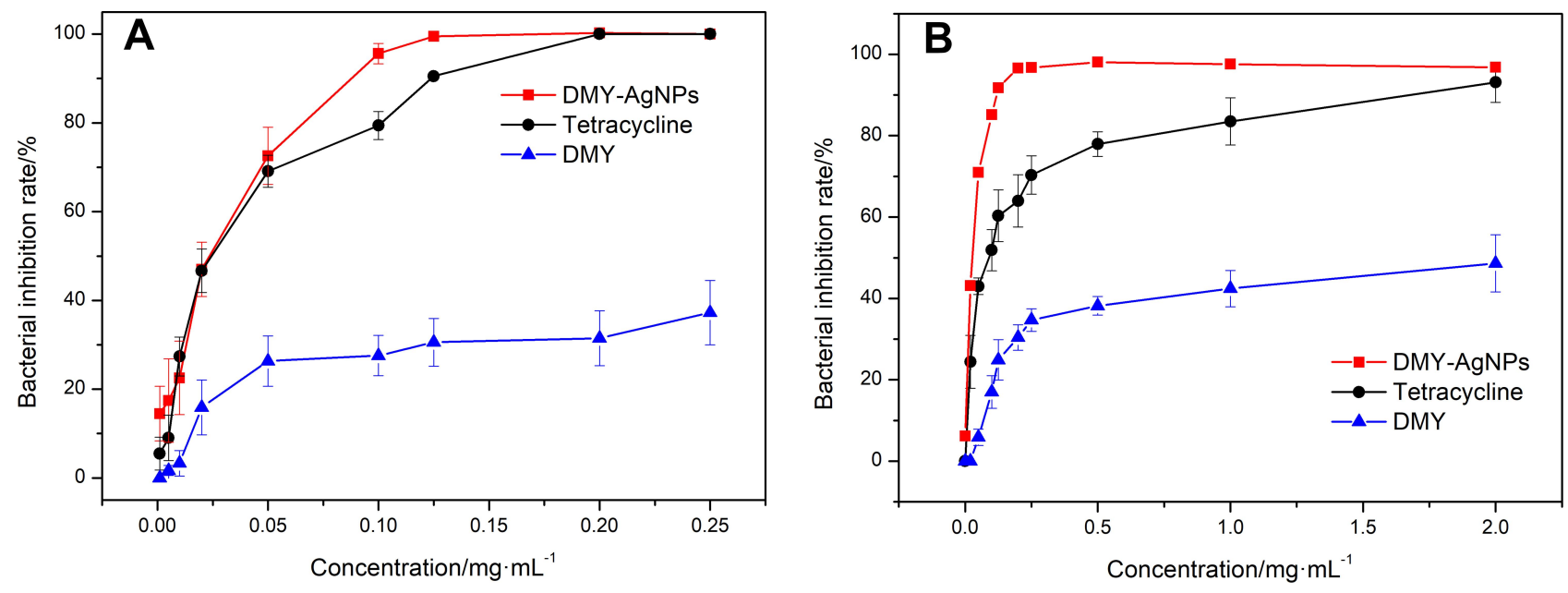

Figure 6 The inhibition rate curves of different samples to bacteria. (A) E. coli. (B) Salmonella. Abbreviation: E. coli, Escherichia coli.

property in colorectal cancer cells, ${ }^{45}$ and to protect myocardial cells from apoptosis. ${ }^{46}$ The nanonization of DMY, as proved in the present study, thus further enhances the anticancer activity, and it provides better chances for the clinical trials.

\section{Conclusion}

The development of DMY-AgNPs with high efficiency, environment-friendly and cost-effective green approach is an important direction of current nanotechnology research and application. Recently biomass has been used as an effective reducing agent and stabilizer for the synthesis of AgNPs. In this study, DMY-mediated AgNPs were successfully synthesized. Compared with previous methods, the present method is simple, non-toxic and environment-friendly. DMY-AgNPs were basically spherical and of moderate size. In addition, biosynthetic AgNPs exhibited DPPH scavenging properties and obvious antibacterial activity against E. coli, and Salmonella, and excellent anticancer activity against Hela, HepG2 and MDA-MB -231 cancer cell lines. These results indicated that the 

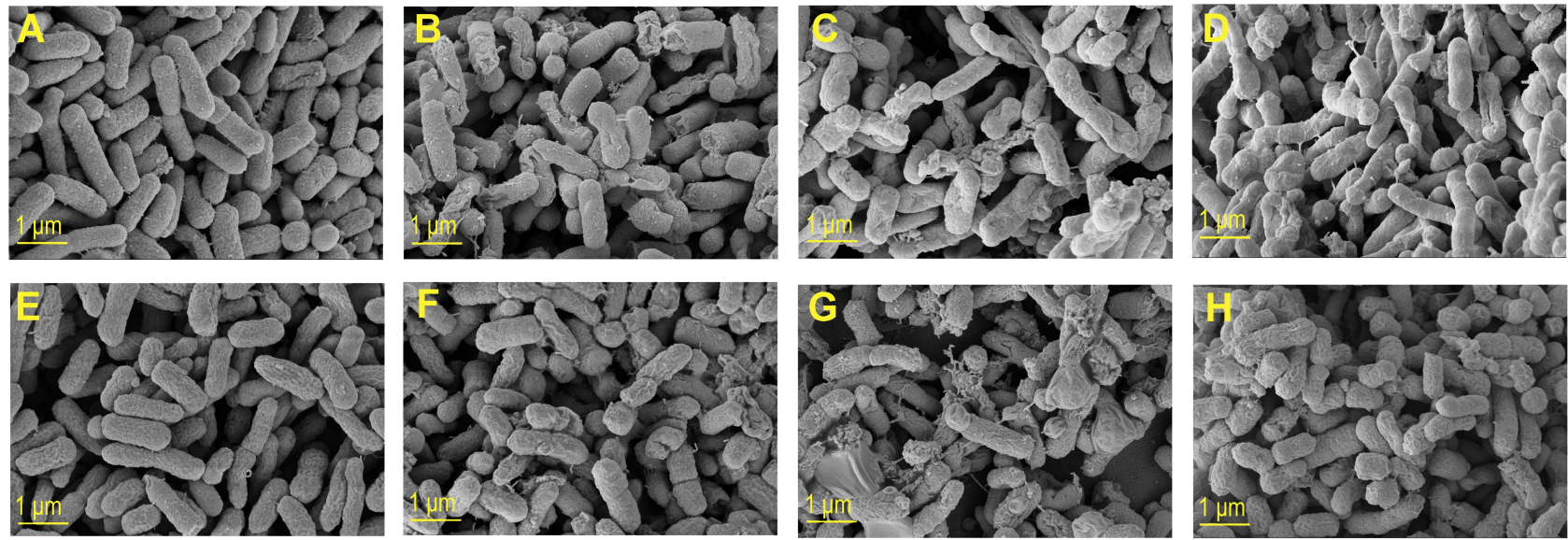

Figure 7 SEM images of bacterial morphology. (A) E. coli. (B) E. coli with DMY. (C) E. coli with DMY-AgNPs. (D) E. coli with tetracycline. (E) Salmonella. (F) Salmonella with DMY. (G) Salmonella with DMY-AgNPs. (H) Salmonella with tetracycline.

Abbreviations: SEM, scanning electron microscopy; E. coli, Escherichia coli; DMY, dihydromyricetin; DMY-AgNPs, dihydromyricetin-mediated silver nanoparticles.
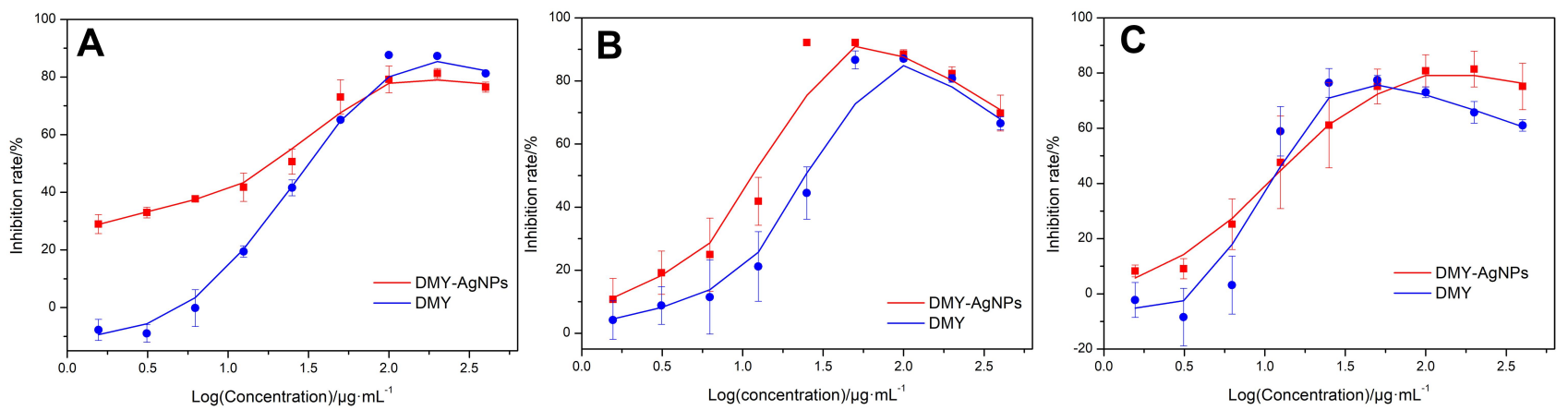

Figure 8 The inhibition rate curves of AgNPs to different cancer cell lines. (A) Hela. (B) HepG2. (C) MDA-MB-23I.

Abbreviations: DMY-AgNPs, dihydromyricetin-mediated silver nanoparticles; HeLa, human cervical cancer cell line; HepG2, human hepatoma cancer cell line; MDA-MB -23 I, breast adenocarcinoma cell line.

DMY-mediated AgNPs could be used as good inhibitors against food-borne bacteria.

\section{Acknowledgments}

We gratefully acknowledge the financial support from the Talented Young Scientist Program (for I.A.), Shandong Province "Double-Hundred Talent Plan" Program, Shandong Province Major Scientific and Technological Innovation Project (2017CXGC1301, 2017CXGC1308). The authors are thankful to Science, Education and Industry Integration Innovation Pilot Project (International Cooperation Project) from Qilu University of Technology (Shandong Academy of Sciences) (2020KJC-GH08).

\section{Disclosure}

The authors report no conflicts of interest in this work.

\section{References}

1. Ong KC, Khoo H-E. Biological effects of myricetin. General Pharmacology. 1997;29(2):121-126. doi:10.1016/S0306-3623(96) 00421-1

2. Zhang J, Chen Y, Luo H, et al. Recent update on the pharmacological effects and mechanisms of dihydromyricetin. Review. Front Pharmacol. 2018;9(1204):01204. doi:10.3389/fphar.2018.01204

3. Ye LY, Wang HJ, Duncan SE, Eigel WN, O'Keefe SF. Antioxidant activities of vine tea (Ampelopsis grossedentata) extract and its major component dihydromyricetin in soybean oil and cooked ground beef. Food Chem. 2015;172:416-422. doi:10.1016/j. foodchem.2014.09.090

4. Gao JH, Liu BG, Ning ZX, Zhao RX, Zhang AY, Wu Q. Characterization and antioxidant activity of flavonoid-rich extracts from leaves of Ampelopsis grossedentata. J Food Biochem. 2009;33 (6):808-820. doi:10.1111/j.1745-4514.2009.00253.x

5. Jia LG, Sheng ZW, Xu WF, et al. Modulation of anti-oxidation ability by proanthocyanidins during germination of Arabidopsis thaliana seeds. Mol Plant. 2012;5(2):472-481. doi:10.1093/mp/ssr089

6. Zuo YZ, Lu YJ, Xu Q, et al. Inhibitory effect of dihydromyricetin on the proliferation of JAR cells and its mechanism of action. Oncol Lett. 2020;20(1):357-363. doi:10.3892/ol.2020.11546 
7. Zeng Y, Hua YQ, Wang W, Zhang H, Xu XL. Modulation of SIRT1-mediated signaling cascades in the liver contributes to the amelioration of nonalcoholic steatohepatitis in high fat fed middle-aged LDL receptor knockout mice by dihydromyricetin. Biochem Pharmacol. 2020;175:113927. doi:10.1016/J.Bcp.2020.113927

8. Yang DF, Yang ZS, Chen L, et al. Dihydromyricetin increases endothelial nitric oxide production and inhibits atherosclerosis through microRNA-21 in apolipoprotein E-deficient mice. $J$ Cell Mol Med. 2020;24(10):5911-5925. doi:10.1111/jcmm.15278

9. Tian YX, Sang HT, Liu MM, et al. Dihydromyricetin is a new inhibitor of influenza polymerase PB2 subunit and influenza-induced inflammation. Microbes Infect. 2020;22(6-7):25 4-262. doi:10.1016/j.micinf.2020.05.021

10. Ni TJ, Lin N, Lu WQ, et al. Dihydromyricetin prevents diabetic cardiomyopathy via miR-34a suppression by activating autophagy. Cardiovasc Drug Ther. 2020;34(3):291-301. doi:10.1007/s10557020-06968-0

11. Wang Y, Pi C, Feng X, Hou Y, Zhao L, Wei Y. The influence of nanoparticle properties on oral bioavailability of drugs. Int J Nanomed. 2020;15:6295-6310. doi:10.2147/IJN.S257269

12. Nilesh S, Raman P. A simple biogenic method for the synthesis of silver nanoparticles using Syngonium podophyllum, an ornamental plant. MGM J Med Sci. 2016;3(3):111-115. doi:10.5005/jp-journals $-10036-1103$

13. Singh P, Kim YJ, Singh H, et al. Biosynthesis, characterization, and antimicrobial applications of silver nanoparticles. Int J Nanomed. 2015;10(1):2567-2577. doi:10.2147/IJN.S72313

14. Ahmed S, Ahmad M, Swami BL, Ikram S. A review on plants extract mediated synthesis of silver nanoparticles for antimicrobial applications: a green expertise. $J A d v$ Res. 2016;7(1):17-28. doi:10.1016/j. jare.2015.02.007

15. Shahbandeh M, Moghadam MT, Mirnejad R, Mirkalantari S, Mirzaei M. The efficacy of $\mathrm{AgNO}_{3}$ nanoparticles alone and conjugated with imipenem for combating extensively drug-resistant Pseudomonas aeruginosa. Int J Nanomed. 2020;15:6905-6916. doi:10.2147/IJN.S260520

16. Abhilash RK, Pandey BD. Microbial synthesis of iron-based nanomaterials-A review. B Mater Sci. 2011;34(2):191-198. doi:10.1007/ s12034-011-0076-6

17. Zhang Y, Cheng X, Zhang Y, Xue X, Fu Y. Biosynthesis of silver nanoparticles at room temperature using aqueous aloe leaf extract and antibacterial properties. Colloids Surf a Physicochem Eng Asp. 2013;423:63-68. doi:10.1016/j.colsurfa.2013.01.059

18. Sarli S, Kalani MR, Moradi A. A potent and safer anticancer and antibacterial Taxus-based green synthesized silver nanoparticle. Int J Nanomed. 2020;15:3791-3801. doi:10.2147/IJN.S251174

19. Kedi PBE, Meva FE, Kotsedi L, et al. Eco-friendly synthesis, characterization, in vitro and in vivo anti-inflammatory activity of silver nanoparticle-mediated Selaginella myosurus aqueous extract. Int J Nanomed. 2018;13:8537-8548. doi:10.2147/IJN.S174530

20. Park TJ, Lee KG, Lee SY. Advances in microbial biosynthesis of metal nanoparticles. Appl Microbiol Biotechnol. 2016;100 (2):521-534. doi:10.1007/s00253-015-6904-7

21. Yu L, Gan X, Zhou D, He F, Zeng S, Hu D. Synthesis and antiviral activity of novel 1, 4-pentadien-3-one derivatives containing a 1, 3, 4-thiadiazole moiety. Molecules. 2017;22(4):658. doi:10.3390/ molecules 22040658

22. Yuan C-G, Huo C, Yu S, Gui B. Biosynthesis of gold nanoparticles using Capsicum annuum var. grossum pulp extract and its catalytic activity. Physica E Low Dimens Syst Nanostruct. 2017;85:19-26. doi:10.1016/j.physe.2016.08.010

23. Yuan CG, Huo C, Gui B, Liu JF, Chen YS. Facile phyto-mediated synthesis of silver nanoparticles using Chinese winter jujube (Ziziphus jujuba Mill. cv. Dongzao) extract and their antibacterial/ catalytic properties. IET Nanobiotechnol. 2017;11(8):973-980. doi:10.1049/iet-nbt.2016.0242
24. Sithara R, Selvakumar P, Arun C, Anandan S, Sivashanmugam P. Economical synthesis of silver nanoparticles using leaf extract of Acalypha hispida and its application in the detection of $\mathrm{Mn}(\mathrm{II})$ ions. $J$ Adv Res. 2017;8(6):561-568. doi:10.1016/j.jare.2017.07.001

25. Zhou Y, Tang RC. Facile and eco-friendly fabrication of AgNPs coated silk for antibacterial and antioxidant textiles using honeysuckle extract. J Photochem Photobiol B. 2018;178:463-471. doi:10.1016/j.jphotobiol.2017.12.003

26. Gurunathan S, Qasim M, Park C, et al. Cytotoxicity and transcriptomic analysis of silver nanoparticles in mouse embryonic fibroblast cells. Int J Mol Sci. 2018;19(11):3618. doi:10.3390/Ijms19113618

27. Vo TS, Le TT, Kim SY, Ngo DH. The role of myricetin from Rhodomyrtus tomentosa (Aiton) Hassk fruits on downregulation of FceRI-mediated mast cell activation. J Food Biochem. 2020;44(3): e13143. doi: $10.1111 /$ jbc. 13143

28. Mendes RA, Almeida SKC, Soares IN, et al. A computational investigation on the antioxidant potential of myricetin 3,4 '-di-O-alphaL-rhamnopyranoside. J Mol Model. 2018;24(6):133. doi:10.1007/ S00894-018-3663-2

29. Cetin-Karaca H, Newman MC. Antimicrobial efficacy of plant phenolic compounds against Salmonella and Escherichia coli. Food Biosci. 2015;11:8-16. doi:10.1016/j.fbio.2015.03.002

30. Silva LN, Da Hora GCA, Soares TA, et al. Myricetin protects Galleria mellonella against Staphylococcus aureus infection and inhibits multiple virulence factors. Sci Rep-Uk. 2017;7:2823. doi:10.1038/S41598-017-02712-1

31. Li G, Wang GZ, Si XS, et al. Inhibition of suilysin activity and inflammation by myricetin attenuates Streptococcus suis virulence. Life Sci. 2019;223:62-68. doi:10.1016/j.1fs.2019.03.024

32. Vivek R, Thangam R, Muthuchelian K, Gunasekaran P, Kaveri K, Kannan S. Green biosynthesis of silver nanoparticles from Annona squamosa leaf extract and its in vitro cytotoxic effect on MCF-7 cells. Process Biochem. 2012;47(12):2405-2410. doi:10.1016/j. procbio.2012.09.025

33. Patra JK, Das G, Baek K-H. Phyto-mediated biosynthesis of silver nanoparticles using the rind extract of watermelon (Citrullus lanatus) under photo-catalyzed condition and investigation of its antibacterial, anticandidal and antioxidant efficacy. $J$ Photochem Photobiol B. 2016;161:200-210. doi:10.1016/j.jphotobiol.2016.05.021

34. Lopez-Miranda JL, Vazquez M, Fletes N, Esparza R, Rosas G. Biosynthesis of silver nanoparticles using a Tamarix gallica leaf extract and their antibacterial activity. Mater Lett. 2016;17 6:285-289. doi:10.1016/j.matlet.2016.04.126

35. Vignesh V, Anbarasi KF, Karthikeyeni S, Sathiyanarayanan G, Subramanian P, Thirumurugan R. A superficial phyto-assisted synthesis of silver nanoparticles and their assessment on hematological and biochemical parameters in Labeo rohita (Hamilton, 1822). Colloids Surf a Physicochem Eng Asp. 2013;439:184-192. doi:10.1016/j. colsurfa.2013.04.011

36. He YQ, Wei FF, Ma ZY, et al. Green synthesis of silver nanoparticles using seed extract of Alpinia katsumadai, and their antioxidant, cytotoxicity, and antibacterial activities. RSC $A d v$. 2017;7(63):39 842-39851. doi:10.1039/c7ra05286c

37. Pandiarajan J, Balaji S, Revathy K, Palanikumar S. Fabrication and validation of silver nanoparticles from cocoon extract of silk worm Bombyx mori. L. Biocatalysis Agricultural Biotechnol. 2018;16:692-705. doi:10.1016/j.bcab.2018.05.017

38. Singh H, Du J, Singh P, Yi TH. Ecofriendly synthesis of silver and gold nanoparticles by Euphrasia officinalis leaf extract and its biomedical applications. Artif Cell Nanomed B. 2018;46(6):1163-1170. doi:10.1080/21691401.2017.1362417

39. Wu YP, Xiao Y, Yue YX, Zhong K, Zhao YL, Gao H. A deep insight into mechanism for inclusion of 2R,3R-dihydromyricetin with cyclodextrins and the effect of complexation on antioxidant and lipid-lowering activities. Food Hydrocolloid. 2020;103:105718. doi:10.1016/j.foodhyd.2020.105718 
40. Teng J, Liu XD, Hu XQ, Zhao YL, Tao NP, Wang MF. Dihydromyricetin as a functional additive to enhance antioxidant capacity and inhibit the formation of thermally induced food toxicants in a cookie model. Molecules. 2018;23(9):2184. doi:10.3390/ Molecules23092184

41. Wu YP, Bai JR, Zhong $\mathrm{K}$, et al. Antibacterial effect of 2R,3R-dihydromyricetin on the cellular functions of Staphylococcus aureus. Biosci Biotech Bioch. 2018;82(1):135-138. doi:10.1080/ 09168451.2017.1413324

42. Wu YP, Bai JR, Zhong K, Huang YN, Gao H. A dual antibacterial mechanism involved in membrane disruption and DNA binding of 2R,3R-dihydromyricetin from pine needles of Cedrus deodara against Staphylococcus aureus. Food Chem. 2017;218:463-470. doi:10.1016/j.foodchem.2016.07.090

43. Xiao XN, Wang F, Yuan YT, Liu J, Liu YZ, Yi X. Antibacterial activity and mode of action of dihydromyricetin from Ampelopsis grossedentata leaves against food-borne bacteria. Molecules. 2019;24 (15):2831. doi:10.3390/Molecules24152831
44. Liu D, Luo J, Wang H, Ding LJ, Zeng XA. Synthesis of dihydromyricetin coated multi-walled carbon nanotubes (MWCNTs) and antibacterial activities. J Nanosci Nanotechnol. 2020;20(10):6148-6154. doi:10.1166/jnn.2020.18002

45. Wang ZY, Sun XT, Feng YY, et al. Dihydromyricetin reverses MRP2-mediated MDR and enhances anticancer activity induced by oxaliplatin in colorectal cancer cells. Anti-Cancer Drug. 2017;28 (3):281-288.

46. Zhu H, Luo PH, Fu YY, et al. Dihydromyricetin prevents cardiotoxicity and enhances anticancer activity induced by adriamycin. Oncotarget. 2015;6(5):3254-3267.

\section{Publish your work in this journal}

The International Journal of Nanomedicine is an international, peerreviewed journal focusing on the application of nanotechnology in diagnostics, therapeutics, and drug delivery systems throughout the biomedical field. This journal is indexed on PubMed Central, MedLine, CAS, SciSearch ${ }^{\mathbb{R}}$, Current Contents ${ }^{\mathbb{R}} /$ Clinical Medicine, $^{-}$
Journal Citation Reports/Science Edition, EMBase, Scopus and the Elsevier Bibliographic databases. The manuscript management system is completely online and includes a very quick and fair peer-review system, which is all easy to use. Visit http://www.dovepress.com/ testimonials.php to read real quotes from published authors. 